\title{
Clinical spectrum and factors impacting outcome of Candida auris: a single center study from Pakistan
}

\author{
Muneeba Ahsan Sayeed, Joveria Farooqi, Kausar Jabeen, Safia Awan and Syed Faisal Mahmood*i]
}

\begin{abstract}
Background: An outbreak of Candida auris began globally in 2014 including Pakistan and since then it has emerged as a nosocomial multi-drug resistant pathogen. The aim of this study was to assess the clinical spectrum and outcome of patients, from a single center in Pakistan, in whom C. auris was isolated.

Methods: A retrospective study was conducted on 92 patients; $\geq 16$ years with at least one culture positive for $C$. auris, at the Aga Khan University Hospital Karachi, Pakistan from Sept 2014-Mar 2017.Demographics, clinical history, management and outcome were studied. A logistic regression model was used to identify the risk factors for mortality.

Results: We identified 92 patients with C. auris (193 isolates), of whom 52.2\% were males. Mean age was $54.14 \pm 20.4$ years. Positive cultures were obtained after a median hospital stay of 14 days. Most patients had a history of surgery (57.6\%), antibiotic use (95.6\%), ICU stay (44.6\%), indwelling lines (88.04\%) and isolation of another multi-resistant organism (52.2\%).Most patients were symptomatic (70.7\%). Amongst these, 38 had candidemia while 27 had non-candidemia infections. Sites of infection included central lines (35), urinary tract (19), peritonitis (4), nosocomial ventriculitis (1), empyema (1), fungal keratitis (1) otitis externa (1) and surgical site (1). Fluconazole resistance was 100\% while 28.5 and $7.9 \%$ were Voriconazole and Amphotericin resistant respectively. Overall crude mortality was $42.4 \%$ while 14 -day mortality was $31.5 \%$. Both infected and colonized cases shared similar mortality (46.2\% vs 33.3\%; $p$-value $=0.25$ ). Among infected cases mortality was high in candidemia compared to non-candidemia ( $60.5 \%$ vs $25.9 \%$ ) in which deaths related to C. auris were $34.2 \%$ vs $22.2 \%$ respectively. On multivariate analysis candidemia (AOR 4.2, 95\% Cl: 1.09-16.49; p-value =0.037) was associated with greater mortality with source control being the only protective factor for mortality (AOR 0.22, 95\% Cl: 0.05-0.92; p-value0.038] while ICU stay, rapidity of blood culture clearance, DM, malignancy and MDR co-infection had no impact.
\end{abstract}

Conclusion: Patients with C.auris from a single center in Pakistan have a wide clinical spectrum with line associated infection being the predominant site of infection. Candidemia leads to high mortality while source control improves outcome.

Keywords: Candida auris, Candidemia, urinary tract infection, Empyema, Colonization, Mortality

\footnotetext{
* Correspondence: faisal.mahmood@aku.edu; phaze97@gmail.com

Section of Infectious Diseases, Department of Medicine, Aga Khan University,

Stadium Rd, Karachi 74800, Pakistan
}

(c) The Author(s). 2019 Open Access This article is distributed under the terms of the Creative Commons Attribution 4.0 International License (http://creativecommons.org/licenses/by/4.0/), which permits unrestricted use, distribution, and reproduction in any medium, provided you give appropriate credit to the original author(s) and the source, provide a link to the Creative Commons license, and indicate if changes were made. The Creative Commons Public Domain Dedication waiver (http://creativecommons.org/publicdomain/zero/1.0/) applies to the data made available in this article, unless otherwise stated. 


\section{Background}

Candida auris has emerged as an antifungal resistant yeast causing invasive infections in nosocomial settings. Improved diagnostics and epidemiological typing techniques are essential to identify and characterize these strains [1, 2]. It was first reported in Japan in 2009 when it was isolated from external ear canal [3]. Later in 2011 it was reported for the first time as human pathogen causing nosocomial fungemia in South Korea and extremes of age with prior history of surgery was identified as a risk factor [4].C. auris was found to be closely related to Candida haemulonii and was reported to be misidentified as C. haemulonii using Vitek 2 YST and Phoenix (BD). There are numerous other misidentifications using the commercially available diagnostic methods. API 20CAUX misidentifies it as Rhodotorula glutinis and C. sake. Vitek 2 (bioMe'rieux) misidentifies it as C. haemulonii, C. lusitaniae and C. famata while MicroScan (Beckman Coulter Pasadena, CA) misidentifies this as C. famata, C. lusitaniae, C. guilliermondii, C. parapsilosis, C. albicans and C. tropicalis [2]. This wrong identification delayed its diagnosis, a finding later endorsed by Kathuria $S$ et al in 2015 [5]. It is notable for its antifungal resistance creating a treatment dilemma [6-8].

In September 2014, Aga Khan University Hospital, Karachi, Pakistan experienced an outbreak of a yeast which was initially identified as Saccharomyces cerevisiae [9]. Because of the unusual antifungal susceptibility pattern of this isolated yeast, these isolates were retested at the Centers for Disease Control and Prevention (CDC), Atlanta, USA for identification and were eventually identified as $C$. auris. Simultaneously this pathogen was reported from India, South Africa and Venezuela and came to lime light [9]. So far, the available literature has emphasized on its methods of identification, antifungal susceptibility and risk factors associated with acquisition. Studies have shown that factors like extremes of age, presence of co-morbidities and prior antifungal therapy are associated with its acquisition while limited data is available on its disease spectrum and outcomes. Therefore, in this study, we have reported its clinical spectrum, outcomes and outcome determining factors among those with invasive infection. The aim of this study was to assess the clinical spectrum and outcome of patients, from a single center in Pakistan, in whom C.auris was isolated.

\section{Methods}

We had conducted a retrospective study of 92 hospitalized patients at the Aga Khan University Hospital (AKUH), a 600-bed tertiary care referral hospital located in Karachi, Pakistan. All patients with $C$. auris positive culture from September 2014-March 2017 were enrolled. Patients aged
$<16$ years or those who did not receive primary treatment at AKUH were excluded.

Cultures were processed by standard operating procedures in use by the microbiology laboratory as part of initial clinical work up. Any yeast from a sterile sample was identified on the basis of germ tube test, appearance on chromogenic medium BiGGY (Oxoid), cycloheximide tolerance, microscopic morphology on Cornmeal Agar (Dalmau method), and profile number on API 20C AUX (bioMe'rieux). If identification was inconclusive (less than $90 \%$ confidence on API), the antifungal susceptibility results for Fluconazole and the API number generated were taken into account. C. auris was presumptively identified by a combination of certain features: resistance to fluconazole, absence of pseudo hyphae on thin Cornmeal Tween80 agar, obtaining profile numbers 2,000,130, $2,000,173,2,102,173,6,102,173$ on API 20C AUX (bioMe'rieux, France). This phenotype had been verified by D1-D2 sequencing of 28S subunit of rDNA performed on the first 15 isolates from our institute sent to the Mycotic Diseases Branch, CDC, Atlanta. There was 100\% concordance between the described phenotype and sequencing results. Antifungal susceptibility was tested using SensititreYeastOne (Trek Diagnostic Systems Ltd., East Grinstead, England) and E-test (bioMe'rieux SA, Marcy l'Etoile, France). Susceptibilities against fluconazole, voriconazole, and amphotericin B were available for all isolates. For an additional 28 isolates MIC values were also available against itraconazole, posaconazole, anidulafungin, micafungin and caspofungin. The susceptibilities were interpreted for Candida species according to CLSI M27-S3 for triazoles and echinocandins and M27-A3 guidelines for amphotericin B $[10,11]$. Briefly, based on these, the isolate was considered resistant to fluconazole if MIC was $\geq 64 \mu \mathrm{g} / \mathrm{ml}$, voriconazole if $\mathrm{MIC}$ was $\geq 4 \mu \mathrm{g} / \mathrm{ml}$, non-susceptible to amphotericin if $\mathrm{MIC}$ was $>1 \mu \mathrm{g} / \mathrm{ml}$, echinocandins if MIC was $>2 \mu \mathrm{g} / \mathrm{ml}$. isolates were considered susceptible if MIC to fluconazole was $\leq 8 \mu \mathrm{g} / \mathrm{ml}$, voriconazole was $\leq 1 \mu \mathrm{g} / \mathrm{ml}$, amphotericin was $\leq 1 \mu \mathrm{g} / \mathrm{ml}$, and echinocandins was $\leq 2 \mu \mathrm{g} / \mathrm{ml}$.

Demographic data including age, gender, date of admission, unit type and a brief history including risk factors (such as diabetes mellitus, malignancy, prior history of surgery, prior antibiotics or antifungal exposure, prior ICU stay, indwelling lines and history of isolation of any other multi-resistant organism), site of infection and outcomes were collected using data collection forms. Data was collected by the primary author only. Patients with $C$. auris in cultures and associated clinical sign and symptoms (including but not limited to fever, site specific symptoms and relevant laboratory parameters such as white blood cells and C-reactive protein) were labeled as symptomatic/infected. On the other hand, patients lacking associated clinical signs and 
symptoms with $C$. auris isolated from non-sterile sites were considered asymptomatic/colonized. In case of multiple positive cultures from the same patient the date of first positive culture was considered as the date of infection/colonization. The site of infection was labeled using CDC definitions [12]. As candida is excluded from CDC's definition for urinary tract infections (UTI), this was defined as clinical sign and symptoms of a UTI with positive urine culture in the presence of urinary WBC and absence of epithelial cells. Asymptomatic candiduria was defined as presence of $C$. auris in urine without clinical sign and symptoms. Standard criteria were used for the definition of diabetes mellitus and malignancy. Use of prior antimicrobials was defined as administration of any antibiotic/ antifungal in last 90 days of positive blood culture. Isolation of other multi-drug resistant (MDR) bacteria before isolating $C$. auris was defined as bacteria that were resistant to more than 3 classes of antibiotics, isolated in last 90 days of positive blood culture for $C$. auris while co infection with MDR bacteria was their isolation within 7 days of positive culture for $C$. auris. Whenever the given antifungal agent was in standard-weight based dosage according to site of infection, and when the isolate was reported as susceptible to it on culture, antifungal therapy was considered appropriate. This is in accordance with our institute's antifungal policy. For all invasive candida cases the first line empiric antifungal therapy was intravenous Amphotericin B deoxycholate $0.75 \mathrm{mg} / \mathrm{kg}$. Lipid formulations of amphotericin B were not used. Voriconazole was considered in cases with deranged renal functions when amphotericin could not be administered. It was given in a dose of $6 \mathrm{mg} / \mathrm{kg} 12$ hourly on day 1 followed by $4 \mathrm{mg} /$ $\mathrm{kg} 12$ hourly. Therapeutic drug monitoring for voriconazole was not available. Early appropriate antifungal therapy was the appropriate antifungal therapy given in $\leq 48$ $\mathrm{h}$ while late appropriate antifungal therapy was started $48 \mathrm{~h}$ after positive culture. Source control was defined as the removal of inciting agent or focus and entailed the removal of central line in case of Central Line Associated Blood Stream Infection (CLABSI), Foley's catheter in Catheter Associated Urinary Tract Infection (CAUTI), exploratory laparotomy in peritonitis, drainage of pus collection and removal of lumbar drain in ventriculitis. Early source control was the removal of inciting agent in $\leq 48 \mathrm{~h}$ while late source control was when it was achieved after $48 \mathrm{~h}$ of positive culture. Only crude mortality rate (CMR) was assessed. Microbiological failure was considered in candidemia patients and was defined as failure to clear blood culture within 4 days while clinical failure was defined as 14-day mortality or microbiological failure. We considered 14-day mortality as there were large number of patients who lost to follow-up during the retrospective chart review. In patients who did not survive, cause of death was also assessed by physicians on clinical and laboratory evidence, and was thought to be related to $C$. auris when other causes of death were excluded and patient had an active $C$. auris infection at that time.

We sought ethical approval from University's Ethics Review Committee (ERC number 4381-MED-ERC-2016). The committee waived the requirement for informed consent. To preserve confidentiality, we coded each patient and removed their original identifications.

The collected data were analyzed through Statistical Package for the Social Sciences (SPSS), version 22.0. For continuous data, mean and standard deviation/ median with interquartile range were reported depending on normality assumption. For categorical data frequency and percentage were reported. In order to assess association of mortality with independent variables Chi square test was applied and $P$ value $<0.05$ was considered significant. Variables for univariate analysis were based on known risk factors of mortality for invasive candidiasis and on the preliminary analysis of our ongoing outbreak study on C. auris $[9,13]$. All statistically significant and clinically relevant variables of univariate analysis were included in a multivariate logistic regression analysis to determine the independent predictive factors of mortality among infective $C$. auris cases. Results were given as odds ratios (OR) and 95\% confidence intervals (95\% CI).

\section{Results}

From 2014 to 2017, 193 C. auris strains were isolated from 92 patients with $16.3 \%$ confirmed as C. auris by D1-D2 sequencing while rest were presumed to be $C$. auris. The number of cases with $C$. auris rose over the study period from 13 patients in the last quarter 2014 to 46 in 2016 (Fig. 1: Epi-curve of C. auris cases). Table 1 describes the baseline characteristics of the patients. The median days from admission to positive culture was 14 (IQR-7-130). Most of the patients were older (median age 55.5 years), were currently or previously admitted to an Intensive Care Unit/ High Dependency Unit (ICU/ HDU), had indwelling lines (central lines and drains), and received antibiotics. One in three patients (39.1\%) had also received antifungals in last 90 days of positive culture. Fifty-two percent had MDR bacteria isolated from different sites prior to C. auris while $31.5 \%$ had a concomitant MDR bacterial infection.

Antifungal susceptibility was checked in isolates from 63 patients $(68.4 \%)$ in which fluconazole resistance was seen in all, voriconazole resistance in $18(28.6 \%)$ and amphotericin resistance seen in $5(7.9 \%)$ patients. Isolates from 3 patients $(4.76 \%)$ were resistant to 2 classes of antifungals (azoles and polyenes) while none were resistant to all classes tested. No echinocandin resistance was detected. 


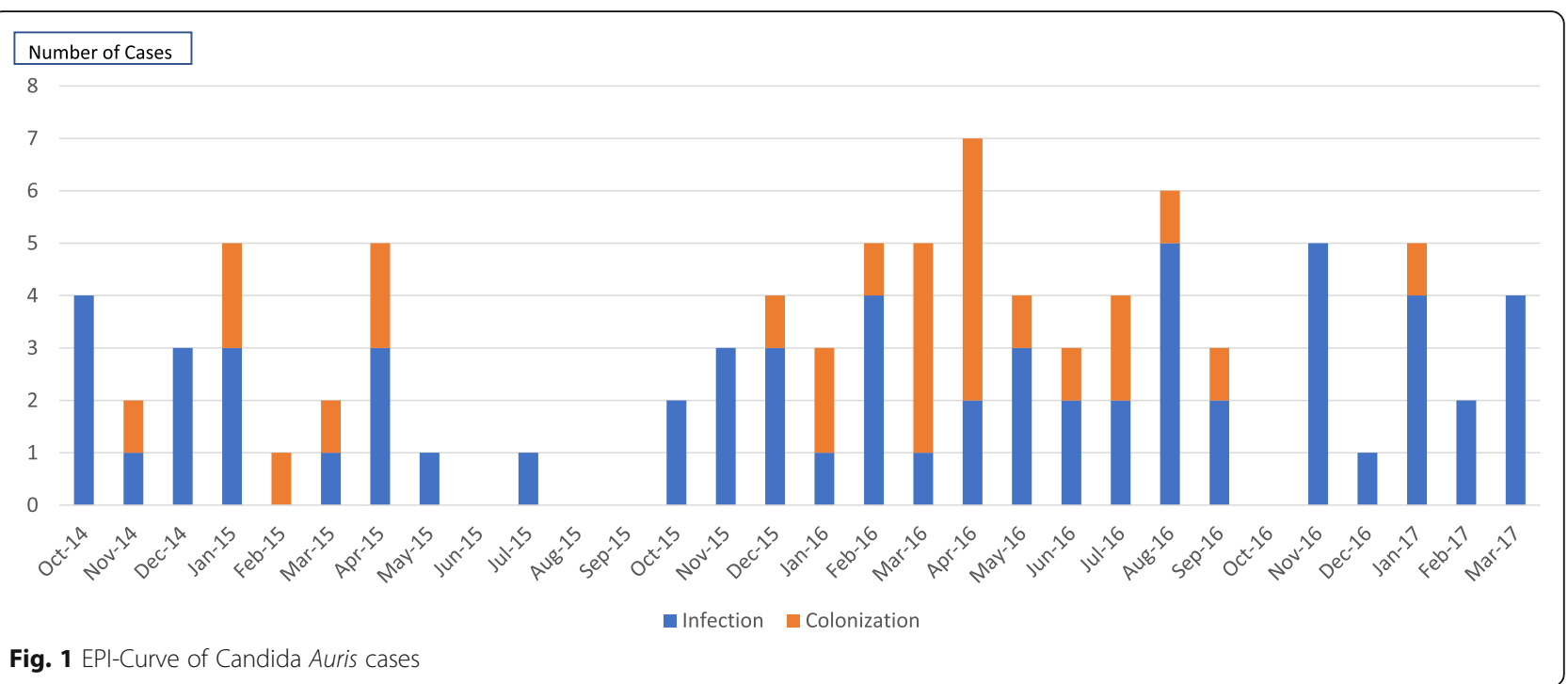

Of the $193 C$. auris strains, majority were isolated from blood (75/193) and urine (83/193). Out of 83 urine specimens 73 were from indwelling catheters, while 10 were mid-stream urine samples. Only 21 patients (22.8\%) isolated C. auris from 2 or more sites.

Of the 92 patients, 65 (70.7\%) were infected while 27 (29.3\%) were colonized with C. auris. Amongst the infected patients, the most common site of infection was the bloodstream accounting for 38 (41.3\%) cases, most of which were CLABSIs $(35,38.4 \%)$. Of the CLABSI patients, 9 (25.71\%) were complicated by seeding to different organs causing endovascular thrombosis or empyema. Source of the fungemia could not be identified in 2 patients. Among non-candidemia patients, UTI was the most commonly seen site infected (19 of 27 patients) with 11 having CAUTI and 8 non-catheter associated UTI.

Of the 65 infected patients, 55 were treated with antifungals, amongst which only 44 patients (67.7\%) received the appropriate antifungal therapy. Early appropriate antifungal therapy was administered to 20 (45.5\%) patients. Amongst the patients who received appropriate antifungal therapy, majority received amphotericin alone (42, 95.5\%) while 2 patients were treated with voriconazole alone. However, 7 patients who initially received amphotericin were later switched to voriconazole. Simultaneous dual antifungal therapy was not given to any patient.

Out of 38 patients with candidemia, 31 (81.57\%) received appropriate antifungal therapy for a mean duration of 18.8 days. Only amphotericin was used to treat candidemia. Early appropriate antifungals were administered in 16 (51.6\%) candidemia patients. However, mortality was higher in those receiving early antifungals (11 of $16,68.7 \%$ ) compared to those who received antifungals late $(7$ of $15 ; 46.7 \%)$. There were 7 patients who did not receive antifungal therapy, of which 2 cleared their candidemia with source control alone. Culture clearance was achieved in 25 out of 27 patients in whom clearance was checked. However, microbiological failure (i.e. lack of clearance within 4 days) was seen in 11 patients. Clearance could not be checked in 10 of the remaining 11 patients due to early mortality.

Amongst the 27 non-candidemia patients, 22 received antifungal, of which 13 were appropriate. In particular, of the 19 patients with UTI, 10 received appropriate antifungal therapy for a mean duration of 12 days. In $8(72.7 \%)$ of the 11 patients with CAUTI, the catheter was removed. Five patients did not receive any antifungal, out of which 2 died within $24 \mathrm{~h}$ of the positive culture. Patients with keratitis received topical and systemic amphotericin while otitis externa was managed with clotrimazole ear drops. Compared to non-candidemia, candidemia patients had a higher mortality rate $(60.5 \%$ vs. $25.9 \% ; p$-value $=0.006)$ and higher 14 -day mortality $(44.7 \%$ vs. $22.2 \%$; p-value $=$ 0.019).

Urine was the most common site of $C$. auris isolation in asymptomatic patients $(21 / 27)$ of whom 17 were catheterized. While the catheter was removed in 10 of these patients, repeat cultures were sent in 2 patients only, both of which were negative. Table 2 compares characteristics and outcomes of infected and colonized C. auris cases. There was no difference in gender, age distribution, location in hospital, co-morbidities, co-infection with MDR bacteria and probability of dying between the two.

The median length of hospital stay in all patients was 25 days (IQR 1-163). The CMR was 39 (42.4\%) in which 19 deaths (48.7\%) were related to $C$. auris. The 14-day mortality was 29 (31.5\%) and the median days in which mortality occurred was 7 (IQR 1-69). 
Table 1 Demographics of 92 patients with Candida auris

\begin{tabular}{|c|c|}
\hline Category & Total $n=92$ \\
\hline Male & $48(52.2 \%)$ \\
\hline \multicolumn{2}{|l|}{ Age group } \\
\hline $16-35$ years & $20(21.7 \%)$ \\
\hline $36-55$ years & $26(28.3 \%)$ \\
\hline $56-75$ years & $30(32.6 \%)$ \\
\hline 76-96 years & $16(17.4 \%)$ \\
\hline \multicolumn{2}{|l|}{ Location in hospital } \\
\hline ICU & $30(32.6 \%)$ \\
\hline Special care & $30(32.6 \%)$ \\
\hline Ward & $27(29.3 \%)$ \\
\hline Outpatient & $5(5.4 \%)$ \\
\hline Diabetes mellitus & $26(28.3 \%)$ \\
\hline Malignancy & $17(18.5 \%)$ \\
\hline Surgery & $53(57.6 \%)$ \\
\hline Abdominal & $12(13 \%)$ \\
\hline Cardiothoracic & $8(8.7 \%)$ \\
\hline Neurosurgery & $14(15.2 \%)$ \\
\hline ENT & $9(9.8 \%)$ \\
\hline Orthopedics & $7(7.6 \%)$ \\
\hline Others & $2(2.17 \%)$ \\
\hline ICU stay in last 30 days & $41(44.6 \%)$ \\
\hline Last ICU stay duration (weeks) $\leq 2$ Weeks & $31(75.6 \%)$ \\
\hline HDU stay in last 30 days & $62(67.4 \%)$ \\
\hline \multicolumn{2}{|l|}{ Last HDU stay duration (weeks) } \\
\hline$\leq 2$ Weeks & $52(56.5 \%)$ \\
\hline Indwelling lines & $81(88.04 \%)$ \\
\hline Antibiotics in last 90 days & $88(95.6 \%)$ \\
\hline Antifungals in last 90 days & $36(39.1 \%)$ \\
\hline Fluconazole & $19(20.7 \%)$ \\
\hline Voriconazole & $3(3.3 \%)$ \\
\hline Amphotericin & $7(7.6 \%)$ \\
\hline$>1$ Antifungal & $7(7.6 \%)$ \\
\hline \multicolumn{2}{|l|}{ Prior antifungal duration (weeks) } \\
\hline$\leq 2$ Weeks & $25(27.2 \%)$ \\
\hline \multicolumn{2}{|l|}{ Isolation of multi-drug resistant bacteria } \\
\hline Prior toC.auris ( $\leq 90$ days) & $48(52.2 \%)$ \\
\hline Coinfection with C.auris & $29(31.5 \%)$ \\
\hline Infected cases & $65(70.7 \%)$ \\
\hline Candidemia & $38(58.5 \%)$ \\
\hline Non-candidemia & $27(41.5 \%)$ \\
\hline UTI & 19 \\
\hline Peritonitis & 3 \\
\hline Nosocomial ventriculitis & 1 \\
\hline Empyema & 1 \\
\hline
\end{tabular}

Table 1 Demographics of 92 patients with Candida auris (Continued)

\begin{tabular}{ll}
\hline Category & Total $n=92(100 \%)$ \\
\hline Surgical Site Infection & 1 \\
Otitis externa & 1 \\
$\quad$ Keratitis & 1 \\
Colonized cases & $27(29.3 \%)$ \\
Asymptomatic candiduria & 21 \\
Central line tip & 4 \\
Ear Swab & 1 \\
Oral Swab & 1 \\
Antifungal resistance ( $n=63)$ & \\
Fluconazole & $63(100 \%)$ \\
Voriconazole & $18(28.57 \%)$ \\
Amphotericin & $5(7.93 \%)$ \\
Mean hospital stay (days) & 30.73 \\
Median hospital stay (days) & $25(1-163)$ \\
Mortality & $39(42.4 \%)$ \\
C auris death & $19(48.7 \%)$ \\
14-day mortality & $29(31.5 \%)$ \\
Mean days in which mortality occurred (days) & 12.41 \\
Median days in which mortality occurred (days) & $7(1-69)$ \\
Clinical failure & $38(41.3 \%)$ \\
Microbiological failure (Candidemia) & $11(40.74 \%)$ \\
\hline
\end{tabular}

Table 3 shows details $C$. auris infective cases and mortality impacting factors. The mean days in which mortality occurred was 5.26 days and 14- day mortality rate was $94.7 \%$.Compared to the infected survivors, these patients were more likely to be older $(56-75$ years, AOR 4.5, 95\%CI:1.1-18.9) and had candidemia (AOR 4.2, 95\%CI: 1.1-16.5). Survivors were also more likely to have had adequate source control (AOR 0.2, 95\%CI: $0.1-0.9)$.

\section{Discussion}

We describe the clinical characteristics and outcomes of patients in whom $C$. auris was isolated in a single center in Pakistan. We found a wide clinical spectrum ranging from asymptomatic patients to invasive disease including candidemia, urinary tract infections, empyema and ventriculitis. Most of the infections occurred in critically ill patients who were admitted for a prolonged period of time.

The crude in-hospital mortality was $42.4 \%$ which is similar to the mortality rate seen in other studies ranging from $35.2-60 \%$ [9, 14]. A total of $31.5 \%$ of the deaths were related to $C$. auris infection, majority of whom died within the first week of acquiring this. This high mortality rate is in contrast with the UK outbreak reported by Schelenz 
Table 2 Comparison of C. auris infected patients with C. auris colonizers

\begin{tabular}{|c|c|c|c|}
\hline Variable & Infection $(n=65)$ & Colonization $(n=27)$ & $p$-value \\
\hline Male & 35 (53.8\%) & $13(48.1 \%)$ & 0.85 \\
\hline \multicolumn{4}{|l|}{ Age group } \\
\hline $16-35$ & $15(23.1 \%)$ & $5(18.5 \%)$ & \\
\hline $36-55$ & $20(30.8 \%)$ & $6(22.2 \%)$ & 0.51 \\
\hline $56-75$ & $21(32.3 \%)$ & $9(33.3 \%)$ & \\
\hline $76-95$ & $9(13.8 \%)$ & $7(25.9 \%)$ & \\
\hline ICU & $23(35.4 \%)$ & 7 (25.9\%) & 0.12 \\
\hline HDU & $24(36.9 \%)$ & $6(22.2 \%)$ & \\
\hline Ward & $11(40.7 \%)$ & $16(24.6 \%)$ & \\
\hline DM & $18(27.7 \%)$ & $8(29.6 \%)$ & 0.85 \\
\hline Malignancy & $13(20 \%)$ & $5(18.5 \%)$ & 0.80 \\
\hline Surgery & $39(60 \%)$ & $14(51.9 \%)$ & 0.61 \\
\hline Co-infection with MDR bacteria & $22(33.8 \%)$ & $7(25.9 \%)$ & 0.45 \\
\hline Appropriate antifungal therapy & $44(67.7 \%)$ & 0 & $<0.001$ \\
\hline Source control & $40(61.5 \%)$ & $7(25.9 \%)$ & $<0.001$ \\
\hline Mortality & $30(46.2 \%)$ & $9(33.3 \%)$ & 0.25 \\
\hline 14-day mortality & $23(35.4 \%)$ & $6(22.2 \%)$ & 0.45 \\
\hline Clinical failure & $24(64.9 \%)$ & $6(22.2 \%)$ & $<0.001$ \\
\hline Alive & 35 (53.8\%) & $18(66.7 \%)$ & \\
\hline C. auris death & 19 (29.2\%) & 0 & \\
\hline Non-auris death & 11 (16.9\%) & $9(33.3 \%)$ & 0.005 \\
\hline
\end{tabular}

Setal where no deaths were directly attributable to $C$. auris [15]. As expected candidemia patients had a higher mortality, though interestingly, antifungal therapy did not make a difference in the mortality. Also, paradoxically we found that mortality was higher in those who received early antifungal treatment; however this is likely reflective of the severity of their illness which prompted rapid initiation of amphotericin which is in contrast to other studies $[6,16]$. Moreover, we could not check if this difference was statistically significant due to the small numbers involved. Mortality was also higher in the older patients, which has been shown in several other studies $[15,17$, 18]. The most important factor impacting outcome was adequate source control. This is in line with established treatment guidelines for treating invasive candidiasis [19]. On the other hand, contrary to other studies, diabetes and malignancy were not associated with mortality in our study, possibly due to small numbers [14].

We found an annual increase in the number of identified cases at our institute. While patients with C.auris are managed with barrier precautions, single room isolation is not possible due to space constraints. Similarly, costs preclude obtaining environmental and patient surveillance cultures. As a result, clonal spread of our strains of C.auris has been documented and highlights the challenges of infection prevention in resource limited countries [9].
Despite skepticism regarding the propensity of $C$. auris to cause urinary tract infection or empyema as stated by CDC, we have found that UTI was the second most common site for symptomatic infection [20]. Moreover, we describe several unusual infection sites including wound, peritoneum, otitis externa, keratitis, ventriculitis and empyema. However, as expected bloodstream infection, in particular CLABSI was the most common infection seen.

The strength of our study is the large number of patients with comprehensive clinical data, giving a better understanding of the spectrum of disease caused by C.auris. Moreover, this is the largest series looking at the gamut of infections caused by the South Asian strain of C.auris in both ICU and non-ICU patients. However, our study has several limitations. Firstly, the data was retrospectively collected leading to potential information bias. Secondly, and perhaps more importantly, not all of our $C$. auris isolates were confirmed by sequencing. However, the characteristic phenotype we have described had $100 \%$ concordance in the 15 strains in which D1-D2 sequencing was performed. Similarly, we were unable to perform genetic antifungal resistance testing. However, of the 15 strains from Pakistan which underwent whole genome sequencing 2 distinct ERG 11 hotspot mutations were identified: Y123F and K143R [9]. 
Table 3 univariate \& multivariate analysis to determine the risk factors for mortality among infective C. auris cases

\begin{tabular}{|c|c|c|c|c|c|c|}
\hline \multirow[t]{2}{*}{ Category } & \multirow[t]{2}{*}{ Died $n=30(100 \%)$} & \multirow[t]{2}{*}{ Survived $n=35$ (100\%) } & \multicolumn{2}{|l|}{ Univariate } & \multicolumn{2}{|l|}{ Multivariable } \\
\hline & & & $\mathrm{OR}^{*}[95 \% \mathrm{Cl}]$ & $P$-value & $\mathrm{AOR}^{\dagger}[95 \% \mathrm{Cl}]$ & $P$-value \\
\hline \multicolumn{7}{|l|}{ Age group } \\
\hline $16-35$ & $5(16.7 \%)$ & $10(28.6 \%)$ & 1.0 & & & \\
\hline $36-55$ & $10(33.3 \%)$ & $10(28.6 \%)$ & $2.0[0.5-7.9]$ & 0.32 & & \\
\hline $56-75$ & $13(43.3 \%)$ & $08(22.9 \%)$ & $3.2[0.8-13]$ & 0.09 & $4.5[1.09-18.9]$ & 0.038 \\
\hline $76-96$ & $02(6.7 \%)$ & 07 (20\%) & $0.5[0.08-3.8]$ & 0.56 & & \\
\hline \multicolumn{7}{|l|}{ Location in hospital } \\
\hline ICU & $14(46.7 \%)$ & 09 (25.7\%) & $3.4[0.8-13]$ & 0.07 & $1.2[0.24-5.8]$ & 0.81 \\
\hline Special Care & $11(36.7 \%)$ & $13(37.1 \%)$ & $1.86[0.5-7.0]$ & 0.35 & & \\
\hline Ward & 05 (16.7\%) & $11(31.4 \%)$ & 1.0 & 0.19 & & \\
\hline Outpatient & 0 & 02 (3.1\%) & & & & \\
\hline Diabetes Mellitus & $11(36.7 \%)$ & $07(20 \%)$ & $2.3[0.7-7.0]$ & 0.13 & & \\
\hline Malignancy & $06(20 \%)$ & $06(17.6 \%)$ & $0.96[0.20-3.2]$ & 0.95 & & \\
\hline Candidemia & $23(76.7 \%)$ & $15(42.9 \%)$ & $4.3[1.4-12]$ & 0.007 & $4.2[1.09-16.49]$ & 0.03 \\
\hline Early blood clearance ( $\leq 72 \mathrm{~h})$ & $02(18.2 \%)$ & $5(35.7 \%)$ & 1.0 & & & \\
\hline Late blood clearance (> 72 h) & $09(81.8 \%)$ & $9(64.3 \%)$ & $2.5[0.38-16.42]$ & 0.34 & & \\
\hline No blood clearance & $02(15.4 \%)$ & $0(0)$ & & & & \\
\hline Appropriate antifungal therapy & $23(76.7 \%)$ & $21(60 \%)$ & $2.2[0.7-6.4]$ & 0.15 & & \\
\hline Early antifungal therapy & $14(46.7 \%)$ & $6(17.1 \%)$ & $4.66[1.2-17.43]$ & 0.02 & $0.84[0.16-4.3]$ & 0.83 \\
\hline Late antifungal therapy & $09(30 \%)$ & $15(42.9 \%)$ & $1.2[0.3-4.09]$ & 0.77 & $4.1[0.94-17.9]$ & 0.06 \\
\hline Source control & $17(42.5 \%)$ & $25(57.5 \%)$ & $0.52[0.18-1.46]$ & 0.2 & $0.22[0.05-0.92]$ & 0.038 \\
\hline Early source control & $07(41.2 \%)$ & $13(56.5 \%)$ & 1.0 & & & \\
\hline Late source control & $10(58.8 \%)$ & $10(43.5 \%)$ & $1.5[0.4-5.3]$ & 0.49 & & \\
\hline Co infection with multidrug resistant bacteria & $13(43.3 \%)$ & 09 (25.7\%) & $2.2[0.77-6.2]$ & 0.13 & $2.7[0.61-12.6]$ & 0.18 \\
\hline
\end{tabular}

\section{*OR, odds ratio}

†AOR, adjusted odds ratio

\section{Conclusion}

In conclusion, C.auris is associated with a wide variety of invasive infections and carries a high mortality rate. Source control is the most effective therapeutic intervention to reduce mortality in these critically ill patients.
Funding

This research did not receive any specific grant from funding agencies in the public, commercial, or not-for-profit sectors.

\section{Availability of data and materials}

The datasets used and/or analyzed during the current study are available from the first author and corresponding author on reasonable request.

\section{Authors' contributions}

SFM, JF and KJ conceived and designed the study; MAS collected data of Candida auris cases; SA and MAS analyzed and interpreted the patient data; MAS drafted the manuscript while SFM, JF and KJ critically analyzed the manuscript for intellectual content; SFM, MAS, JF and KJ were major contributors in writing the manuscript and all authors read and approved the final manuscript.

\section{Authors' information}

Syed Faisal Mahmood, M.B.B.S, DABIM, DABIM (ID), Associate Professor, Section of Infectious Diseases, Department of Medicine, Aga Khan University, Karachi, Pakistan.

Muneeba Ahsan Sayeed, M.B.B.S, FCPS (Medicine), FCPS (ID), ID Fellow, Section of Infectious Diseases, Department of Medicine, Aga Khan University, Karachi, Pakistan.

Joveria Farooqi, M.B.B.S, FCPS (Microbiologist), MSc (Epidemiology and Biostatistics), Assistant Professor, Section of Microbiology, Department of Pathology, Aga Khan University, Karachi, Pakistan. 
Kausar Jabeen, M.B.B.S, FCPS (Microbiologist), MSc (Medical Mycology), Associate Professor, Section of Microbiology, Department of Pathology, Aga Khan University, Karachi, Pakistan

Safia Awan, MSc Statistics, Senior Instructor Research, Department of Medicine, Aga Khan University, Karachi, Pakistan.

\section{Ethics approval and consent to participate}

We sought ethical approval from Aga Khan University's Ethics Review Committee (ERC number 4381-MED-ERC-2016). The committee waived the requirement for informed consent. To preserve confidentiality, we coded each patient and removed their original identifications.

\section{Consent for publication}

Not applicable

\section{Competing interests}

The authors declare that they have no competing interests.

\section{Publisher's Note}

Springer Nature remains neutral with regard to jurisdictional claims in published maps and institutional affiliations.

Received: 22 November 2018 Accepted: 17 April 2019

Published online: 06 May 2019

\section{References}

1. Cortegiani A, Misseri G, Fasciana T, Giammanco A, Giarratano A, Chowdhary A. Epidemiology, clinical characteristics, resistance, and treatment of infections by Candida auris. J Intensive Care. 2018;6:69.

2. Jeffery-Smith A, Taori SK, Schelenz S, Jeffery K, Johnson EM, Borman A, et al. Candida auris: a Review of the Literature. Clin Microbiol Rev. 2017;31(1).

3. Satoh K, Makimura K, Hasumi Y, Nishiyama Y, Uchida K, Yamaguchi H. Candida auris sp. nov., a novel ascomycetous yeast isolated from the external ear canal of an inpatient in a Japanese hospital. Microbiol Immunol. 2009;53(1):41-4.

4. Lee WG, Shin JH, Uh Y, Kang MG, Kim SH, Park KH, et al. First three reported cases of nosocomial fungemia caused by Candida auris. J Clin Microbiol. 2011;49(9):3139-42.

5. Kathuria S, Singh PK, Sharma C, Prakash A, Masih A, Kumar A, et al. Multidrug-resistant Candida auris misidentified as Candida haemulonii: characterization by matrix-assisted laser desorption ionization-time of flight mass spectrometry and DNA sequencing and its antifungal susceptibility profile variability by Vitek 2, CLSI broth microdilution, and Etest method. J Clin Microbiol. 2015;53(6):1823-30.

6. Chowdhary A, Anil Kumar V, Sharma C, Prakash A, Agarwal K, Babu R, et al. Multidrug-resistant endemic clonal strain of Candida auris in India. Eur J Clin Microbiol Infect Dis. 2014;33(6):919-26.

7. Sarma S, Kumar N, Sharma S, Govil D, Ali T, Mehta Y, et al. Candidemia caused by amphotericin B and fluconazole resistant Candida auris. Indian J Med Microbiol. 2013;31(1):90-1.

8. Magobo RE, Corcoran C, Seetharam S, Govender NP. Candida aurisassociated candidemia, South Africa. Emerg Infect Dis. 2014;20(7):1250-1.

9. Lockhart SR, Etienne KA, Vallabhaneni S, Farooqi J, Chowdhary A, Govender $N P$, et al. Simultaneous emergence of multidrug-resistant Candida auris on 3 continents confirmed by whole-genome sequencing and epidemiological analyses. Clin Infect Dis. 2017;64(2):134-40.

10. CLSI. Reference method for broth dilution antifungal susceptibility testing of yeasts; Third International Supplement. In: CLSI document M27-S3. Wayne, PA: Clinical and Laboratory Standards Institute; 2008.

11. CLSI. Reference method for broth dilution antifungal susceptibility testing of yeasts; Third International Supplement. In: CLSI document M27-A3: Wayne, PA, Clinical and Laboratory Standards Institute; 2008.

12. CDC/NHSN Surveillance Definitions for Specific Types of Infections January 2016. Accessed from https://www.cdc.gov/nhsn/PDFs/pscManual/ 17pscNosInfDef_current.pdf.

13. Cortés JA, Reyes P, Gómez CH, Cuervo Sl, Rivas P, Casas CA, Sánchez R. Clinical and epidemiological characteristics and risk factors for mortality in patients with candidemia in hospitals from Bogotá, Colombia. Braz J Infect Dis. 2014;18(6):631-7.
14. Morales-López SE, Parra-Giraldo CM, Ceballos-Garzón A, Martínez HP, Rodríguez GJ, Álvarez-Moreno CA, et al. Invasive infections with multidrugresistant yeast Candida auris, Colombia. Emerg Infect Dis. 2017;23(1):162-4.

15. Schelenz S, Hagen F, Rhodes UL, Abdolrasouli A, Chowdhary A, Hall A, et al. First hospital outbreak of the globally emerging Candida auris in a European hospital. Antimicrob Resist Infect Control. 2016;5:35.

16. Todd B. Clinical alert: Candida Auris. Am J Nurs. 2017;117(4):53-5.

17. Chowdhary A, Sharma C, Duggal S, Agarwal K, Prakash A, Singh PK, Jain S, et al. New clonal strain of Candida auris, Delhi, India. Emerg Infect Dis. 2013; 19:1670-3.

18. Ruiz Gaitán AC, Moret A, López Hontangas JL, Molina JM, Aleixandre López Al, Cabezas AH, et al. Nosocomial fungemia by Candida auris: first four reported cases in continental Europe. Rev IberoamMicol. 2017;34(1):23-7.

19. Pappas PG, Kauffman CA, Andes DR, Clancy CJ, Marr KA, Ostrosky-Zeichner $L$, et al. Executive summary: clinical practice guideline for the Management of Candidiasis: 2016 update by the Infectious Diseases Society of America. Clin Infect Dis. 2016;62(4):409-17.

20. Centers for Disease Control and Prevention. 2018. https://www.cdc.gov/ fungal/candida-auris/candida-auris-qanda.html.

\section{Ready to submit your research? Choose BMC and benefit from:}

- fast, convenient online submission

- thorough peer review by experienced researchers in your field

- rapid publication on acceptance

- support for research data, including large and complex data types

- gold Open Access which fosters wider collaboration and increased citations

- maximum visibility for your research: over $100 \mathrm{M}$ website views per year

At BMC, research is always in progress.

Learn more biomedcentral.com/submissions 